PROCEEDINGS OF THE

AMERICAN MATHEMATICAL SOCIETY

Volume 126, Number 3, March 1998, Pages 779-789

S 0002-9939(98)04125-2

\title{
MINKOWSKI'S INEQUALITY FOR TWO VARIABLE DIFFERENCE MEANS
}

\author{
LÁSZLÓ LOSONCZI AND ZSOLT PÁLES
}

(Communicated by J. Marshall Ash)

Abstract. We study Minkowski's inequality

$D_{a b}\left(x_{1}+x_{2}, y_{1}+y_{2}\right) \leq D_{a b}\left(x_{1}, y_{1}\right)+D_{a b}\left(x_{2}, y_{2}\right) \quad\left(x_{1}, x_{2}, y_{1}, y_{2} \in \mathbb{R}_{+}\right)$

and its reverse where $D_{a b}$ is the difference mean introduced by Stolarsky. We give necessary and sufficient conditions (concerning the parameters $a, b$ ) for the inequality above (and for its reverse) to hold.

\section{INTRODUCTION}

Let $a, b \in \mathbb{R}$ be two distinct nonzero real numbers. Cauchy's mean value theorem, applied to the functions $t \rightarrow t^{a}, t \rightarrow t^{b}$ on a subinterval $[y, x]$ of the positive real numbers $\mathbb{R}_{+}$, gives that the number

$$
\xi=\left(\frac{b\left(x^{a}-y^{a}\right)}{a\left(x^{b}-y^{b}\right)}\right)^{\frac{1}{a-b}}
$$

lies between $y$ and $x$. Thus $D_{a b}(x, y)=\xi$ is a mean value of $x$ and $y$. This mean value was first defined by Stolarsky [20] who showed that $D_{a b}(x, y)$ can be extended continuously to the domain $R=\left\{(a, b, x, y) \mid a, b \in \mathbb{R}, x, y \in \mathbb{R}_{+}\right\}$. This extension is given by

$$
D_{a b}(x, y)= \begin{cases}\left(\frac{b\left(x^{a}-y^{a}\right)}{a\left(x^{b}-y^{b}\right)}\right)^{\frac{1}{a-b}} & \text { if } a b(a-b)(x-y) \neq 0, \\ \left(\frac{x^{a}-y^{a}}{a(\ln x-\ln y)}\right)^{\frac{1}{a}} & \text { if } a(x-y) \neq 0, b=0, \\ \left(\frac{b(\ln x-\ln y)}{x^{b}-y^{b}}\right)^{-\frac{1}{b}} & \text { if } b(x-y) \neq 0, a=0, \\ \exp \left(-\frac{1}{a}+\frac{x^{a} \ln x-y^{a} \ln y}{x^{a}-y^{a}}\right) & \text { if } b(x-y) \neq 0, a=b, \\ \sqrt{x y} & \text { if } x-y \neq 0, a=b=0, \\ x & \text { if } x-y=0 .\end{cases}
$$

$D_{a b}(x, y)$ is called the difference mean or Stolarsky's mean of $x$ and $y$. The power mean $M_{a}(x, y)$ with exponent $a$ can be obtained as $D_{2 a a}(x, y)$; thus $D_{21}, D_{00}$, and

Received by the editors April 3, 1996 and, in revised form, September 3, 1996.

1991 Mathematics Subject Classification. Primary 26D15, 26 D07.

Key words and phrases. Difference means, Minkowski's inequality.

Research of the first author supported by Kuwait University Grant SM 145 and research of the second author by the Hungarian National Foundation for Scientific Research (OTKA), Grant No. T-016846. 
$D_{-2-1}$ are the arithmetic, geometric, and harmonic means respectively. $D_{01}$ and $D_{11}$ are called logarithmic and identric means, respectively.

The comparison problem

$$
D_{a b}(x, y) \leq D_{c d}(x, y)
$$

for difference means on $\mathbb{R}_{+}$(i.e. if in (2) $x, y \in \mathbb{R}_{+}$) was solved by Leach and Sholander [11]. Páles [15] gave a new proof for this result. In [16] Páles solved the comparison problem (2) on any subinterval $(\alpha, \beta)$ of $\mathbb{R}_{+}$. Several particular inequalities involving $D_{a b}$ and its special cases were dealt with by Alzer [2], Brenner [3], Brenner and Carlson [4], Burk [5], Carlson [6], Dodd [7], Leach and Sholander [10], Lin [13], Pittinger [17], [18], Sándor [22], Seiffert [23], [24], Stolarsky [20], and Székely [25]. Neuman [14] studied multivariable weighted logarithmic means; Leach and Sholander [12] dealt with difference means with several variables.

Some of these means have applications in electrostatics [1], [19], in heat conductions and chemical problems [26]. Of particular recent interest is the occurrence of these means in signal processing theory in connection with time-frequency distributions. See [8], especially pp. 17-20, 34-36, 38 .

The aim of this paper is to find necessary and sufficient conditions for Minkowski's inequality

$$
D_{a b}\left(x_{1}+x_{2}, y_{1}+y_{2}\right) \leq D_{a b}\left(x_{1}, y_{1}\right)+D_{a b}\left(x_{2}, y_{2}\right) \quad\left(x_{1}, x_{2}, y_{1}, y_{2} \in \mathbb{R}_{+}\right)
$$

(and its reverse inequality) to hold.

\section{REsults}

Our results can be formulated in three theorems.

Theorem 1. The inequality (3) holds if and only if

$$
a+b \geq 3, \quad \min \{a, b\} \geq 1 .
$$

Theorem 2. The inequality

$$
D_{a b}\left(x_{1}+x_{2}, y_{1}+y_{2}\right) \geq D_{a b}\left(x_{1}, y_{1}\right)+D_{a b}\left(x_{2}, y_{2}\right) \quad\left(x_{1}, x_{2}, y_{1}, y_{2} \in \mathbb{R}_{+}\right)
$$

holds if and only if

$$
a+b \leq 3, \quad \min \{a, b\} \leq 1 .
$$

Figure 1 shows the domain of points $(a, b)$ satisfying condition (4) and (6).

The following numerical examples illustrate the typical inequalities that can be obtained from Theorem 1 and Theorem 2. (For the sake of simplicity, we assume that $\left(x_{1}-y_{1}\right)\left(x_{2}-y_{2}\right)\left(x_{1}+x_{2}-y_{1}-y_{2}\right) \neq 0$ in the inequalities below.)

The points $(2,2),(3,1),(4,2)$ satisfy $(4)$. Thus, Theorem 1 yields the following inequalities (after multiplying by some constant factor):

$$
\begin{gathered}
\left(\frac{\left(x_{1}+x_{2}\right)^{\left(\left(x_{1}+x_{2}\right)^{2}\right)}}{\left(y_{1}+y_{2}\right)^{\left(\left(y_{1}+y_{2}\right)^{2}\right)}}\right)^{\frac{1}{\left(x_{1}+x_{2}\right)^{2}-\left(y_{1}+y_{2}\right)^{2}}} \leq\left(\frac{x_{1}^{\left(x_{1}^{2}\right)}}{y_{1}^{\left(y_{1}^{2}\right)}}\right)^{\frac{1}{x_{1}^{2}-y_{1}^{2}}}+\left(\frac{x_{2}^{\left(x_{2}^{2}\right)}}{y_{2}^{\left(y_{2}^{2}\right)}}\right)^{\frac{1}{x_{2}^{2}-y_{2}^{2}}}, \\
\sqrt{\left(x_{1}+x_{2}\right)^{2}+\left(x_{1}+x_{2}\right)\left(y_{1}+y_{2}\right)+\left(y_{1}+y_{2}\right)^{2}} \\
\leq \sqrt{x_{1}^{2}+x_{1} y_{1}+y_{1}^{2}}+\sqrt{x_{2}^{2}+x_{2} y_{2}+y_{2}^{2}} \\
\sqrt{\left(x_{1}+x_{2}\right)^{2}+\left(y_{1}+y_{2}\right)^{2}} \leq \sqrt{x_{1}^{2}+y_{1}^{2}}+\sqrt{x_{2}^{2}+y_{2}^{2}} .
\end{gathered}
$$




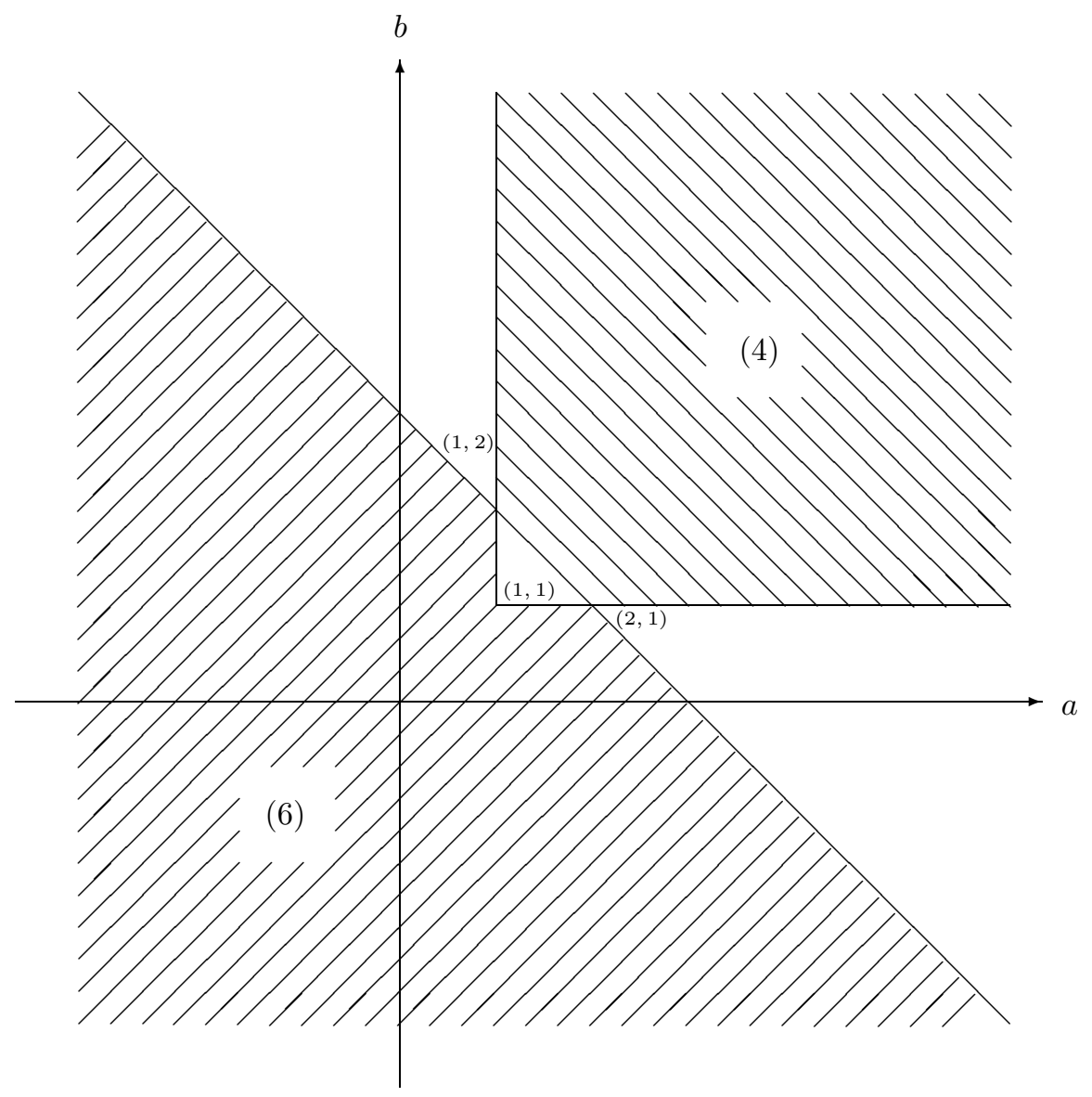

FIGURE 1

(The last inequality is equivalent to the two dimensional Cauchy-Schwarz inequality.) Analogously, the points $(0,0),(2,-1),(1,0)$ satisfy $(6)$. Therefore, by Theorem 2 , we obtain

$$
\begin{aligned}
\sqrt{\left(x_{1}+x_{2}\right)\left(y_{1}+y_{2}\right)} & \geq \sqrt{x_{1} y_{1}}+\sqrt{x_{2} y_{2}} \\
\sqrt[3]{\left(x_{1}+x_{2}\right)^{2}\left(y_{1}+y_{2}\right)+\left(x_{1}+x_{2}\right)\left(y_{1}+y_{2}\right)^{2}} & \geq \sqrt[3]{x_{1}^{2} y_{1}+x_{1} y_{1}^{2}}+\sqrt[3]{x_{2}^{2} y_{2}+x_{2} y_{2}^{2}}, \\
\frac{x_{1}+x_{2}-y_{1}-y_{2}}{\ln \left(x_{1}+x_{2}\right)-\ln \left(y_{1}+y_{2}\right)} & \geq \frac{x_{1}-y_{1}}{\ln x_{1}-\ln y_{1}}+\frac{x_{2}-y_{2}}{\ln x_{2}-\ln y_{2}} .
\end{aligned}
$$

Since the domains given by (4) and (6) have just two common points $(1,2)$ and $(2,1)$, we have the following

Corollary 1. The identity

(7) $D_{a b}\left(x_{1}+x_{2}, y_{1}+y_{2}\right)=D_{a b}\left(x_{1}, y_{1}\right)+D_{a b}\left(x_{2}, y_{2}\right) \quad\left(x_{1}, x_{2}, y_{1}, y_{2} \in \mathbb{R}_{+}\right)$

holds if and only if

$$
(a, b)=(1,2) \quad \text { or } \quad(a, b)=(2,1)
$$


Concerning equality in (3) and (6), we have

Theorem 3. Let $(a, b) \in \mathbb{R}^{2},(a, b) \neq(1,2),(a, b) \neq(2,1)$. Suppose that (3) (or

(5)) holds. Then equality is valid in (3) (or in (5)) if and only if

$$
\frac{x_{1}}{x_{2}}=\frac{y_{1}}{y_{2}} \text {. }
$$

\section{ProOfS}

The properties of $D_{a b}$ are summarized in

Lemma 1. Let, for $(a, b, x, y) \in R, d(a, b, x, y):=D_{a b}(x, y)$. Then we have

(i) $\quad d(a, b, x, y)=d(b, a, x, y)=d(a, b, y, x)$,

(ii) $\quad d(a, b, t x, t y)=t d(a, b, x, y)$,

(iii) $\quad d \in C^{\infty}(R)$

for all $(a, b, x, y) \in R, t \in \mathbb{R}_{+}$.

Proof. Properties (i) and (ii) follow immediately from the definition of $d$ and from (1).

To prove that $d$ is infinitely many times differentiable in all of its variables we need an integral form of $d$. Let

$$
F(a, x, y):=\ln \int_{0}^{1}(u x+(1-u) y)^{a-1} d u \quad\left(a \in \mathbb{R} ; x, y \in \mathbb{R}_{+}\right) .
$$

Since

$$
0<\min \{x, y\} \leq u x+(1-u) y \leq \max \{x, y\},
$$

the integrand in (9) is a positive function having continuous partial derivatives of any order with respect to $a, x, y$ on $\mathbb{R} \times \mathbb{R}_{+} \times \mathbb{R}_{+}$. Thus both $F$ and $f$ defined by

$$
f(a, x, y)=\frac{\partial}{\partial a} F(a, x, y)
$$

are in $C^{\infty}\left(\mathbb{R} \times \mathbb{R}_{+} \times \mathbb{R}_{+}\right)$.

It is easy to check that for all $x, y \in \mathbb{R}_{+}$

$$
D_{a b}(x, y)= \begin{cases}\left(\frac{\exp F(a, x, y)}{\exp F(b, x, y)}\right)^{\frac{1}{a-b}}=\exp \frac{F(a, x, y)-F(b, x, y)}{a-b} & \text { if } a-b \neq 0, \\ \exp f(a, x, y) & \text { if } a-b=0 .\end{cases}
$$

Using the identity

$$
\int_{0}^{1} f(v b+(1-v) a, x, y) d v= \begin{cases}\frac{F(a, x, y)-F(b, x, y)}{a-b} & \text { if } a-b \neq 0 \\ f(a, x, y) & \text { if } a-b=0\end{cases}
$$

we have

$$
d(a, b, x, y)=D_{a b}(x, y)=\exp \int_{0}^{1} f(v b+(1-v) a, x, y) d v \quad(a, b, x, y) \in R,
$$

which shows that the function $d$ is in $C^{\infty}(R)$ proving (iii).

Lemma 2. Let $M: \mathbb{R}_{+} \times \mathbb{R}_{+} \rightarrow \mathbb{R}$ be an arbitrary homogeneous function of degree one (i.e. $M(t x, t y)=t M(x, y)$ holds for all $\left.x, y, t \in \mathbb{R}_{+}\right)$. Then Minkowski's inequality

$$
M\left(x_{1}+x_{2}, y_{1}+y_{2}\right) \leq M\left(x_{1}, y_{1}\right)+M\left(x_{2}, y_{2}\right) \quad\left(x_{1}, x_{2}, y_{1}, y_{2} \in \mathbb{R}_{+}\right)
$$


(or its reverse inequality) holds if and only if the function $m$ defined by $m(u)=$ $M(u, 1)$ is a convex (or concave) function on $\mathbb{R}_{+}$.

If $m$ is a strictly convex (or strictly concave) function on $\mathbb{R}_{+}$, then equality occurs in (11) (or in its reverse inequality) if and only if

$$
\frac{x_{1}}{x_{2}}=\frac{y_{1}}{y_{2}}
$$

Proof. Since $M(x, y)=y M\left(\frac{x}{y}, 1\right)=y m\left(\frac{x}{y}\right)$, we can write (11) as

$$
m\left(\frac{y_{1}}{y_{1}+y_{2}} \frac{x_{1}}{y_{1}}+\frac{y_{2}}{y_{1}+y_{2}} \frac{x_{2}}{y_{2}}\right) \leq \frac{y_{1}}{y_{1}+y_{2}} m\left(\frac{x_{1}}{y_{1}}\right)+\frac{y_{2}}{y_{1}+y_{2}} m\left(\frac{x_{2}}{y_{2}}\right)
$$

or, with the notations $\frac{x_{1}}{y_{1}}=u \in \mathbb{R}_{+}, \frac{x_{2}}{y_{2}}=v \in \mathbb{R}_{+}, \frac{y_{1}}{y_{1}+y_{2}}=\lambda \in(0,1)$,

$$
m(\lambda u+(1-\lambda) v) \leq \lambda m(u)+(1-\lambda) m(v),
$$

which is exactly the convexity of $m$. If $m$ is strictly convex, then equality stands in (12) or in (11) if and only if $u=v$ (see e.g. [9]). The proof for the reverse inequality is similar.

The next lemma is well-known again (see e.g. [9]).

Lemma 3. Let $I$ be an open subinterval of $\mathbb{R}$ and $g: I \rightarrow \mathbb{R}$ be a function of class $C^{2}(I)$. The function $g$ is convex [concave] if and only if $g^{\prime \prime}(x) \geq 0(x \in I)$ $\left[g^{\prime \prime}(x) \leq 0(x \in I)\right]$.

If $g^{\prime \prime}(x)>0(x \in I \backslash A)\left[g^{\prime \prime}(x)<0(x \in I \backslash A)\right]$, where $A$ is a finite subset of $I$, then $g$ is strictly convex [concave], that is, in the convexity inequality

$$
g(\lambda u+(1-\lambda) v) \leq \lambda g(u)+(1-\lambda) g(v)
$$

[or in its reverse] equality holds if and only if $\lambda \in\{0,1\}, u, v \in I$ or $\lambda \in[0,1], u=$ $v \in I$.

Proof of Theorem 1. By Lemmas 1-3 (3) holds if and only if $m_{a b}^{\prime \prime}(u) \geq 0 \quad(u \in$ $\mathbb{R}_{+}$), where $m_{a b}$ is the continuous (moreover $C^{\infty}$ ) extension of the function

$$
\begin{aligned}
D_{a b}(u, 1)=\left(\frac{b\left(u^{a}-1\right)}{a\left(u^{b}-1\right)}\right)^{\frac{1}{a-b}}=\sqrt{u} & \left(\frac{b \sinh (a \ln \sqrt{u})}{a \sinh (b \ln \sqrt{u})}\right)^{\frac{1}{a-b}} \\
& \left(u \in \mathbb{R}_{+}, a b(a-b)(u-1) \neq 0\right)
\end{aligned}
$$

to $a, b \in \mathbb{R}, u \in \mathbb{R}_{+}$. Using logarithmic differentiation, we obtain after some calculation that

$$
\begin{aligned}
\frac{4 u^{2} m_{a b}^{\prime \prime}(u)}{m_{a b}(u)}= & \frac{a^{2}(1-a+b)}{(a-b)^{2}}\left(\operatorname{coth}^{2} a s-1\right)+\frac{b^{2}(1+a-b)}{(a-b)^{2}}\left(\operatorname{coth}^{2} b s-1\right) \\
& -\frac{2 a b}{(a-b)^{2}}(\operatorname{coth} a s \operatorname{coth} b s-1),
\end{aligned}
$$

where $s=\ln \sqrt{u} \in \mathbb{R}$ and $a b(a-b) s \neq 0$. We need to write (13) in other forms, too.

The multiplication of (13) by

$$
2 \frac{\sinh ^{2} a s}{(a s)^{2}} \frac{\sinh ^{2} b s}{(b s)^{2}}
$$

removes the singularity $s=0$ in (13). Applying the addition formula of cosh and the identity $\cosh ^{2} x-\sinh ^{2} x=1$ we obtain

$$
\begin{aligned}
G_{a b}(s) & :=\frac{8 u^{2} m_{a b}^{\prime \prime}(u)}{m_{a b}(u)} \frac{\sinh ^{2} a s}{(a s)^{2}} \frac{\sinh ^{2} b s}{(b s)^{2}} \\
& =\frac{2 a^{2}(1-a+b) \sinh ^{2} b s+2 b^{2}(1+a-b) \sinh ^{2} a s-4 a b \sinh a s \sinh b s \cosh (a-b) s}{(a b(a-b) s)^{2}} .
\end{aligned}
$$


Using the half-angle formula for $\sinh ^{2}$, the "product into sum" formula for hyperbolic functions and the identities $a^{2}(1-a+b)-a b=a(1-a)(a-b), b^{2}(1+a-b)-a b=$ $-b(1-b)(a-b)$ we can write

$$
G_{a b}(s)=\frac{(1-a)(\cosh 2 b s-1)}{a b^{2}(a-b) s^{2}}-\frac{(1-b)(\cosh 2 a s-1)}{a^{2} b(a-b) s^{2}}+\frac{\cosh 2(a-b) s-1}{a b(a-b)^{2} s^{2}} .
$$

From (14) we easily obtain the Taylor series of $G_{a b}$ :

$$
G_{a b}(s)=\sum_{n=2}^{\infty} g_{n}(a, b) \frac{(2)^{2 n} s^{2 n-2}}{(2 n) !} \quad(s \in \mathbb{R}),
$$

where

$$
g_{n}(a, b)=\frac{(1-a) b^{2 n-1}-(1-b) a^{2 n-1}+(a-b)^{2 n-1}}{a b(a-b)} \quad(n=2,3, \ldots) .
$$

One can easily see that $g_{n}$ is a polynomial of $a, b$; hence it can be defined for all $a, b \in \mathbb{R}$. Since the factors $\frac{\sinh ^{2} a s}{(a s)^{2}}, \frac{\sinh ^{2} b s}{(b s)^{2}}$ have the limit 1 at $a=0, b=0, s=0$, the series development (15) of $G_{a b}$ is valid for all $a, b, s \in \mathbb{R}$.

Now we are ready to prove that the conditions (4) are necessary for (3) to hold. By Lemma 3, (3) implies that $G_{a b}(s) \geq 0$. Hence

$$
g_{2}(a, b)=\lim _{s \rightarrow 0} \frac{G_{a b}(s)}{s^{2}} \geq 0 .
$$

This gives the condition

$$
g_{2}(a, b)=\frac{(1-a) b^{3}-(1-b) a^{3}+(a-b)^{3}}{a b(a-b)}=a+b-3 \geq 0 .
$$

Multiplying $G_{a b}$ by a suitable factor and then taking its limit at $s=\infty$, we can get another necessary condition for (3). From (14) one can see that $G_{a b}(s) s^{2}$ is a linear combination of four cosh terms (the fourth one being cosh $0 s$ ). The next lemma will help to find the suitable factor for the limiting process.

Lemma 4. Let

$$
\begin{aligned}
H(s) & :=\sum_{k=1}^{4} A_{k}\left(a_{1}, a_{2}, a_{3}, a_{4}\right) \cosh a_{k} s \quad(s \in \mathbb{R}), \\
c & :=\max \left\{\left|a_{1}\right|,\left|a_{2}\right|,\left|a_{3}\right|,\left|a_{4}\right|\right\},
\end{aligned}
$$

where $A_{k}$ are arbitrary functions of the real variables $a_{1}, a_{2}, a_{3}, a_{4}$. Assume that $\left|a_{1}\right|,\left|a_{2}\right|,\left|a_{3}\right|,\left|a_{4}\right|$ are pairwise distinct numbers. Then

$$
\lim _{s \rightarrow \infty} 2 e^{-c s} H(s)=A_{l}\left(a_{1}, a_{2}, a_{3}, a_{4}\right) \quad \text { if } \quad c=\left|a_{l}\right| .
$$

Proof. Our statement easily follows from the limit relation

$$
\lim _{s \rightarrow \infty} 2 e^{-c s} \cosh \alpha s= \begin{cases}1 & \text { if } c=|\alpha| \\ 0 & \text { if } c>|\alpha| .\end{cases}
$$

Let us return to the proof of necessity. By (i) of Lemma 1, we may assume, without restricting the generality, that $a \geq b$. This and (17) show that the point 
$(a, b)$ is either in the first or in the fourth quadrant. We claim that the latter case: $a>0, b<0$ is not possible. Assuming it, we would get from (13), that

$$
\lim _{u \rightarrow \infty} \frac{4 u^{2} m_{a b}^{\prime \prime}(u)}{m_{a b}(u)}=\frac{4 a b}{(a-b)^{2}} \geq 0
$$

which is impossible. Therefore $a \geq 0, b \geq 0$.

Case 1: $a>b>0$.

Since $\max \{2|a|, 2|b|, 2|a-b|, 0\}=2|a|=2 a$, by Lemma 4, we get that

$$
\lim _{s \rightarrow \infty} 2 s^{2} G_{a b}(s) e^{-2 a s}=-\frac{1-b}{a^{2} b(a-b)} \geq 0
$$

which implies that

$$
b \geq 1 .
$$

The inequalities (17) and (18) give exactly the condition (4) in Case 1.

Case 2: $a>b=0$.

From (14) we obtain for $a \neq 0$ that

$$
G_{a 0}(s)=\lim _{b \rightarrow 0} G_{a b}(s)=\frac{2 a^{2}(1-a) s^{2}+(a+1)(\cosh 2 a s-1)-2 a s \sinh 2 a s}{a^{4} s^{2}}
$$

and

$$
\lim _{s \rightarrow \infty} 2 s^{2} G_{a 0}(s) e^{-2 a s}=-\infty,
$$

which shows that (3) cannot hold if $a>b=0$.

Thus, if $a \geq b \geq 0$, then the necessary conditions for (3) are: (17) and (18). Extending the region given by (17),(18) symmetrically to the line $a=b$, we get exactly (4).

Next we prove that the conditions (4) are sufficient for (3) to hold. For symmetry reasons, we may assume again that $a \geq b \geq 0$. The region $S$ determined by (4) and $a \geq b \geq 0$ can be written as $S_{1} \cup S_{2}$, where

$$
\begin{aligned}
& S_{1}=\left\{(a, b) \mid 3 \leq a+b, 1 \leq b<\frac{a}{2}\right\}, \\
& S_{2}=\left\{(a, b) \mid 3 \leq a+b, \frac{a}{2} \leq b \leq a\right\} .
\end{aligned}
$$

We shall show by induction that, for $(a, b) \in S$,

$$
g_{n}(a, b) \geq 0 \quad(n=2,3, \ldots) .
$$

This implies that $G_{a b}(s) \geq 0$ if $(a, b) \in S$ and then, by Lemmas 1-3, (3) follows. We have

$$
g_{2}(a, b)=a+b-3 \geq 0 \quad \text { if } \quad(a, b) \in S
$$

and for all $(a, b) \in \mathbb{R}^{2}$

$$
g_{n+1}(a, b)=(a-b)^{2} g_{n}(a, b)+h_{n}(a, b) \quad(n=1,2, \ldots),
$$

where $g_{1}(a, b)=0$ and

$$
h_{n}(a, b)=\frac{a^{2 n-2}(b-1)(2 a-b)+b^{2 n-2}(a-1)(a-2 b)}{a-b} \quad(n=1,2, \ldots) .
$$

It is clear that $h_{n}$ is a polynomial in $a$ and $b$.

If $(a, b) \in S_{1}$, then $a \geq 2, a-2 b>0,2 a-b=a+(a-b) \geq 0$. Therefore both terms of $h_{n}$ are nonnegative and so is $h_{n}(a, b)$. By (19) this implies by induction that $g_{n}(a, b) \geq 0$. 
If $(a, b) \in S_{2}$, then $2 b-a \geq 0, a \geq b \geq 1, a+b \geq 3$ and in the identity

$$
h_{n+1}(a, b)=a^{2} h_{n}(a, b)+(a-1)(2 b-a)(a+b) b^{2 n-2}
$$

the second term is nonnegative since each of its factors are such. On the other hand, $h_{1}(a, b)=a+b-1 \geq 0$; therefore, by induction $h_{n}(a, b) \geq 0$ and by (19), we get $g_{n}(a, b) \geq 0$.

This completes the proof of Theorem 1 .

Proof of Theorem 2. The conditions (6) are necessary. We may suppose that $a \geq b$. We are going to prove that (4) implies $b \leq \min \{1,3-a\}$. By Lemma 2 and Lemma 3 , $G_{a b}(s) \leq 0(s \in \mathbb{R})$. Thus

$$
\lim _{s \rightarrow 0} \frac{G_{a b}(s)}{s^{2}}=g_{2}(a, b)=a+b-3 \leq 0 .
$$

If $a \geq b>0$ we can derive another necessary condition.

Case 1: $a>b>0$.

Similarly to the proof of Theorem 1, we get, by Lemma 4 , that

$$
\lim _{s \rightarrow \infty} 2 s^{2} G_{a b}(s) e^{-2 a s}=-\frac{1-b}{a^{2} b(a-b)} \leq 0 ;
$$

therefore,

$$
b \leq 1
$$

Case 2: $a=b>0$.

From (14), we get (for $a \neq 0$ ) that

$$
G_{a a}(s)=\lim _{b \rightarrow a} G_{a b}(s)=\frac{2 a^{2} s^{2}-(2 a-1)(\cosh 2 a s-1)-2 a(1-a) s \sinh 2 a s}{a^{4} s^{2}} ;
$$

hence

$$
\lim _{s \rightarrow \infty} 2 s G_{a a}(s) e^{-2 a s}=-2 a(1-a) \leq 0 .
$$

This gives the same necessary condition $b=a \leq 1$, completing the proof of necessity.

To prove the sufficiency of (6), we assume again that $a \geq b$ and decompose the region $T$ determined by (6) and $a \geq b$ into the union of the following six sets:

$$
\begin{aligned}
& T_{1}=\{(a, b) \mid a \leq 0, b \leq 0, a \geq b\}, \\
& T_{2}=\left\{(a, b) \mid a \geq 1, b \leq \frac{a}{2}, 0 \leq a+b \leq 3\right\}, \\
& T_{3}=\left\{(a, b) \mid 0<a<1,-a \leq b \leq \frac{a}{2}\right\}, \\
& T_{4}=\left\{(a, b) \mid a \geq 1, \frac{a}{2}<b \leq 1\right\}, \\
& T_{5}=\left\{(a, b) \mid 0<a<1, \frac{a}{2}<b \leq a\right\}, \\
& T_{6}=\{(a, b) \mid a>0, b<-a\} .
\end{aligned}
$$

We shall prove that for $(a, b) \in T$ the coefficients $g_{n}(a, b)$ are nonpositive. Hence $G_{a b}(s) \leq 0$, which implies (4). Let $h_{n}(a, b)$ be defined by (20). Then we have (19).

If $(a, b) \in T_{1} \cup T_{2}$, then

$$
h_{1}(a, b)=a+b-3 \leq 0
$$

and in the identity

$$
h_{n+1}(a, b)=a^{2} h_{n}(a, b)+(a-1)(2 b-a)(a+b) b^{2 n-2}
$$

the second term of the right-hand side is nonpositive. In $T_{1}$, we have $a-1<$ $0, a+b \leq 0, b \leq a \leq \frac{a}{2}$, while in $T_{2}$ the inequalities $a-1 \geq 0, a+b \geq 0, b \leq \frac{a}{2}$ 
hold. Hence, by induction, it follows that $h_{n}(a, b) \leq 0(n=1,2, \ldots)$ and by (19), we get, again by induction, the required inequality

$$
g_{n}(a, b) \leq 0 \quad(n=2,3, \ldots) .
$$

If $(a, b) \in T_{3} \cup T_{4}$ then both terms of

$$
h_{n}(a, b)=\frac{a^{2 n-2}(b-1)(2 a-b)}{a-b}+\frac{b^{2 n-2}(a-1)(a-2 b)}{a-b} \quad(n=1,2, \ldots)
$$

are nonpositive. In $T_{3} \cup T_{4}$ we have $b-1 \leq 0,2 a-b \geq 0$, and thus the first term of $h_{n}$ is nonpositive. In $T_{3}$ we have $a-1 \leq 0, a-2 b \geq 0$ while in $T_{4}$ the inequalities $a-1 \geq 0, a-2 b<0$ hold; hence in both cases the second term of $h_{n}$ is nonpositive. Using (19) we get, by induction, the inequality (25).

If $(a, b) \in T_{5}$, then in the identity

$$
g_{n+1}(a, b)=a^{2} g_{n}(a, b)+\frac{a+b}{a}\left[(a-1) b^{2 n-2}+(b-2 a)(a-b)^{2 n-3}\right](n=1,2, \ldots)
$$

both terms in the bracket are nonpositive, the factor in front of the bracket is positive, and hence, by induction, we get (25).

If $(a, b) \in T_{6}$, then $-b \geq a>0$,

$$
(a-b)^{2 n-1} \geq a^{2 n-1}+(-b)^{2 n-1} .
$$

Hence

$$
\begin{aligned}
& (1-a) b^{2 n-1}-(1-b) a^{2 n-1}+(a-b)^{2 n-1} \geq \\
& (a-1)(-b)^{2 n-1}-(1-b) a^{2 n-1}+a^{2 n-1}+(-b)^{2 n-1}= \\
& a(-b)^{2 n-1}+b a^{2 n-1}=a b\left[a^{2 n-2}-b^{2 n-2}\right] \geq 0
\end{aligned}
$$

and by $a b \leq 0$

$$
g_{n}(a, b)=\frac{(1-a) b^{2 n-1}-(1-b) a^{2 n-1}+(a-b)^{2 n-1}}{a b(a-b)} \leq 0 \quad(n=2,3, \ldots),
$$

proving (25).

Thus, we have shown that (25) holds on the whole set $T$ which proves the sufficiency of conditions (6).

Proof of Theorem 3. Suppose that (3) holds. Then (4) is also true by Theorem 1.

If $b>3-a$ in (4), then $g_{2}(a, b)=a+b-3>0$, the other coefficients $g_{n}(n=$ $3,4, \ldots)$ are nonnegative, and hence $G_{a b}(s)>0$ for $s \neq 0$ which implies the strict convexity of $m_{a b}$ on $\mathbb{R}_{+}$. By Lemma 2 equality holds in (3) if and only if (8) is satisfied.

If $b=3-a$ in (4), then $g_{2}(a, b)=a+b-3=0$. By assumption $a \neq 1, a \neq 2$; thus, we have $1<a<2, b=3-a$. We claim that for these pairs $(a, b)$ the coefficient $g_{3}$ is positive. A simple calculation shows that

$$
g_{3}(a, b)=(a+b)^{3}-5(a+b)^{2}-2 a b(a+b)+15 a b,
$$

and therefore,

$$
g_{3}(a, 3-a)=-9(a-1)(a-2)>0 \text { if } 1<a<2 .
$$

The other coefficients $g_{n}(a, b)(n=4,5, \ldots)$ are nonnegative in the region (4); hence $G_{a b}(s)>0$ for $s \neq 0$, which implies the strict convexity of $m_{a b}$ on $\mathbb{R}_{+}$. By Lemma 2, equality occurs in (3) if and only if (8) holds. 
If (5) holds, then by Theorem 2 (6) is true. If $a+b<3$ in (6), then $g_{2}(a, b)<$ $0, g_{n}(a, b) \leq 0(n=3,4, \ldots)$. If $a+b=3$ in $(6)$, then either $a<1$, or $a>2$, in both cases $g_{3}(a, 3-a)=-9(a-1)(a-2)<0$, and $g_{n}(a, 3-a) \leq 0(n=4,5, \ldots)$. Therefore $G_{a b}(s)>0, m_{a b}$ is strictly concave and, by Lemma 2, equality occurs in (5) if and only if (8) is valid.

\section{REFERENCES}

1. W. H. Adams, Heat transmission, McGraw-Hill, New York, 1954.

2. H. Alzer, Bestmögliche Abschätzungen für spezielle Mittelwerte, Univ. u Novom Sadu Zb. Rad. Prirod.-Mat. Fak. Ser. Mat., 23(1) 1993, 331-346. MR 95m:26028

3. J. Brenner, A unified treatment and extension of some means of classical analysis I. Comparison theorems, J. Combin. Inform System Sci., 3 1978, 175-199. MR 80d:26017

4. J. Brenner and B. C. Carlson, Homogeneous mean values: Weights and asymptotics, J. Math. Anal. Appl., 123 1987, 265-280. MR 88g:26023b

5. F. Burk, By all means, Amer. Math. Monthly, 92 1985, 50.

6. B. C. Carlson, The logarithmic mean, Amer. Math. Monthly, 79 1972, 615-618. MR 46:1985

7. E. L. Dodd, Some generalization of the logarithmic mean and of similar means of two variates which become indeterminate when the two variates are equal, Ann. Math. Statist., 12 1941, 422-428. MR 3:170e

8. P. Flandrin and P. Gonçalvès, Geometry of affine time-frequency distributions, Applied and Computational Harmonic Anal., 3 (1996), 10-39. MR 96:94002

9. J.-B. Hiriart-Uruty and C. Lemarechál, Convex Analysis and Minimization Algorithms I, Springer Verlag, Heidelberg, 1993. MR 95m:90001

10. E. Leach and M. Sholander, Extended mean values, Amer. Math. Monthly, 85 1978, 84-90. MR 58:22428

11. E. Leach and M. Sholander, Extended mean values II, J. Math. Anal. Appl., 92 1983, 207-223. MR 85b:26007

12. E. Leach and M. Sholander, Multi-variable extended mean values, J. Math. Anal. Appl., 104 1984, 390-407. MR 86b:26033

13. T. P. Lin, The power mean and the logarithmic mean, Amer. Math. Monthly, 81 1974, 879-883. MR 50:7449

14. E. Neuman, The weighted logarithmic mean, J. Math. Anal. Appl., 188 1994, 885-900. MR 95k:26018

15. Zs. Páles, Inequalities for differences of powers, J. Math. Anal. Appl., 131 1988, 271-281. MR 89f: 26023

16. Zs. Páles, Comparison of two variable homogeneous means, General Inequalities 6. Proc. 6th Internat. Conf. Math. Res. Inst. Oberwolfach, Birkhäuser Verlag Basel, 1992, pp. 59-69. MR 94b:26016

17. A. O. Pittenger, The symmetric, logarithmic, and power means, Univ. Beograd. Publ. Elektrotehn. Fak., Ser. Mat. Fiz. No. 681 1980, 19-23. MR 83i:26016b

18. A. O. Pittenger, The logarithmic mean in $n$ variables, Amer. Math. Monthly, 92 1985, 99-104. MR 86h:26012

19. G. Pólya and G. Szegö, Isoperimetric inequalities in mathematical physics, Princeton Univ. Press, Princeton, N. J., 1951. MR 13:270d

20. K. B. Stolarsky, Generalizations of the logarithmic mean, Math. Mag., 48 1975, 87-92. MR 50:10186

21. K. B. Stolarsky, The power and generalized logarithmic means, Amer. Math. Monthly, 87 1980, 545-548. MR 82g:26029

22. J. Sándor, On certain inequalities for means, J. Math. Anal. Appl., 189 1995, 602-606. MR 95k:26025

23. H. Seiffert, Ungleichungen für einen bestimmten Mittelwert, Nieuw Arch. Wisk., 13 1995, 195-198. MR 96h:26025

24. H. Seiffert, Ungleichungen für elementare Mittelwerte, Arch. Math. (Basel), 64 1995, 129131. MR 95j:26026 
25. G. Székely, A classification of means, Ann. Univ. Sci. Budapest. Eötvös Sect. Math., 18 1975, 129-133. MR 54:7723

26. K. Tettamanti, G. Sárkány, D. Králik and R. Somfai, Über die Annäherung logarithmischer Funktionen durch algebraische Funktionen, Period. Polytech. Chem. Engrg., 14 1970, 99-111.

Department of Mathematics and Computer Science, Kuwait University, P.O.Box 5969 SAFAT, 13060 KuWAIT

E-mail address: losonczi@math-1.sci.kuniv.edu.kw

Institute of Mathematics, Lajos Kossuth University, H-4010 Debrecen, Pf. 12, HunGARY

E-mail address: pales@math.klte.hu 